\title{
ERRATUM
}

Kosuke Itoh

\section{Personality research with non-human primates: theoretical formulation and methods}

Published online: 6 August 2004

(C) Japan Monkey Centre and Springer-Verlag 2004

\section{Primates (2002) 43:249-261}

In the second line of the "Introduction" section of this article, "inter-species" should be corrected to "intraspecies".

K. Itoh

Center for Integrated Human Brain Science,

Brain Research Institute, University of Niigata,

1-757 Asahimachi-dori, Niigata 951-8585, Japan

E-mail: itoh@bri.niigata-u.ac.jp

Tel.: + 81-25-2270680

Fax: $+81-25-2270822$ 